\title{
Rare Combination of Adverse Effects Associated With Lamotrigine Treatment
}

\author{
Mustafa Ceylanª, ${ }^{\mathrm{a}}$ Onur Ceylan ${ }^{\mathrm{b}}$
}

\begin{abstract}
Lamotrigine (LTG) is an antiepileptic drug which is widely used in treatment of epilepsy. It is effective as an adjunctive treatment of refractory partial seizures and idiopathic generalized epilepsy in adults and children. However, patients are subject to some adverse effects with ongoing medication of LTG. Rare events associated with LTG medication such as hematological abnormalities and skin lesions have utmost clinical importance and often cause drug withdrawal. We present a case of a 23-year-old male who had leukopenia, thrombocytopenia and rash associated with LTG treatment. Clinician must be aware of serious side effects of LTG especially at the beginning of the treatment. Serious adverse effects such as leukopenia, thrombocytopenia and rash should be considered along with the other insignificant complaints such as nausea, dizziness and malaise.
\end{abstract}

Keywords: Lamotrigine; Rash; Leukopenia; Thrombocytopenia

\section{Introduction}

Lamotrigine (LTG) is an antiepileptic drug which is widely used in treatment of epilepsy. The mechanism of LTG is based on inhibition of dihydrofolate reductase enzyme which causes blocking the influx of sodium ions. This blockage results in reduced excitation by excess glutamate and stabilizes neuronal membranes [1]. It is effective as an adjunctive treatment of refractory partial seizures and idiopathic generalized epilepsy in adults and children [2]. However, patients are subject to some adverse effects with ongoing medication of LTG. These side effects are often transient and clinically insignificant. However, rare events associated with LTG medication such as hematological abnormalities and skin lesions have utmost clinical importance and often cause drug

Manuscript submitted May 17, 2017, accepted June 5, 2017

aDeparment of Neurology, Medical Faculty of Ataturk University, Erzurum, Turkey

${ }^{b}$ Deparment of Pathology, Regional Education and Research Hospital, Erzurum, Turkey

${ }^{\mathrm{c} C}$ Corresponding Author: Mustafa Ceylan, Osmangazi M. Esat S. Sarıbugdaylar Sitesi B-Blok No: 3-16, Palandöken Erzurum, Turkey.

Email: drmuson16@hotmail.com

doi: https://doi.org/10.14740/jnr442w withdrawal. Hereby, we present a case of LTG induced leukopenia, thrombocytopenia and rash which occurred during the fifth week of treatment. To our knowledge, this report is unique regarding the presence of such combination of side effects.

\section{Case Report}

A 23-year-old male patient was admitted to outpatient clinic with nausea, dizziness, malaise and fever which started 3 days ago. He had a history of mild mental retardation and seizures since childhood. He had a medication of levetiracetam $(3,000 \mathrm{mg} /$ day $)$ and LTG $(150 \mathrm{mg} /$ day $)$ at the time of admission. Medication records revealed that he was on medication of valproic acid (2,500 mg/day) and levetiracetam $(2,250 \mathrm{mg} /$ day) for nearly 13 years. This medication was revised by his neurologist 5 weeks ago due to new symptoms associated with long term usage of valproic acid. Recorded symptoms were tremor, alopecia and weight gain. Serum valproic acid level was $91 \mu \mathrm{g} / \mathrm{mL}$ (normal range: $50-100 \mu \mathrm{g} / \mathrm{mL}$ ) at that time. Valproic acid was decreased to $1,500 \mathrm{mg} /$ day, levetiracetam was increased to $3,000 \mathrm{mg} /$ day and LTG with a dose of $25 \mathrm{mg} /$ day was added to therapy. After deploying this treatment regimen, electroencephalography (EEG) showed spike-and-wave discharges. Thus, valporic acid dose was gradually decreased and ceased in 2 weeks; levetiracetam was continued at same dose; LTG dose was gradually increased to $150 \mathrm{mg} /$ day (increment: $25 \mathrm{mg} / 5$ days) in 5 weeks.

Physical and neurologic examination revealed no abnormalities. He had a body temperature of $39^{\circ} \mathrm{C}$, blood pressure of 130/85 mm Hg and heart rate of 98 beats per minute. Laboratory test results were in normal range by means of biochemical tests. Complete blood cell count revealed low white blood cells $\left(2.32 \times 10^{3} / \mu \mathrm{L}\right.$, normal range: $\left.3.7-10.1 \times 10^{3} / \mu \mathrm{L}\right)$, low platelet $\left(126 \times 10^{3} / \mu \mathrm{L}\right.$, normal range: $\left.150-450 \times 10^{3} / \mu \mathrm{L}\right)$ and low neutrophil $\left(1.2 \times 10^{3} / \mu \mathrm{L}\right.$, normal range: $1.4-6.5 \times 10^{3} /$ $\mu \mathrm{L})$. Serologic tests for hepatitis A, B and C, rubella, HIV, Epstein-Barr virus, toxoplasma and cytomegalovirus were normal. Gruber-Widal test and tests of anti-nuclear antibody and anti-dsDNA were also normal. There were no traces of blast cell on peripheral blood smear. Abdominal ultrasonography was normal which was performed to exclude intra-abdominal organ pathologies. EEG was normal which was performed at the time of admission.

On the second day of admission, patient developed maculo-papular rash on skin of face, trunk and limbs (Fig. 1). Skin 

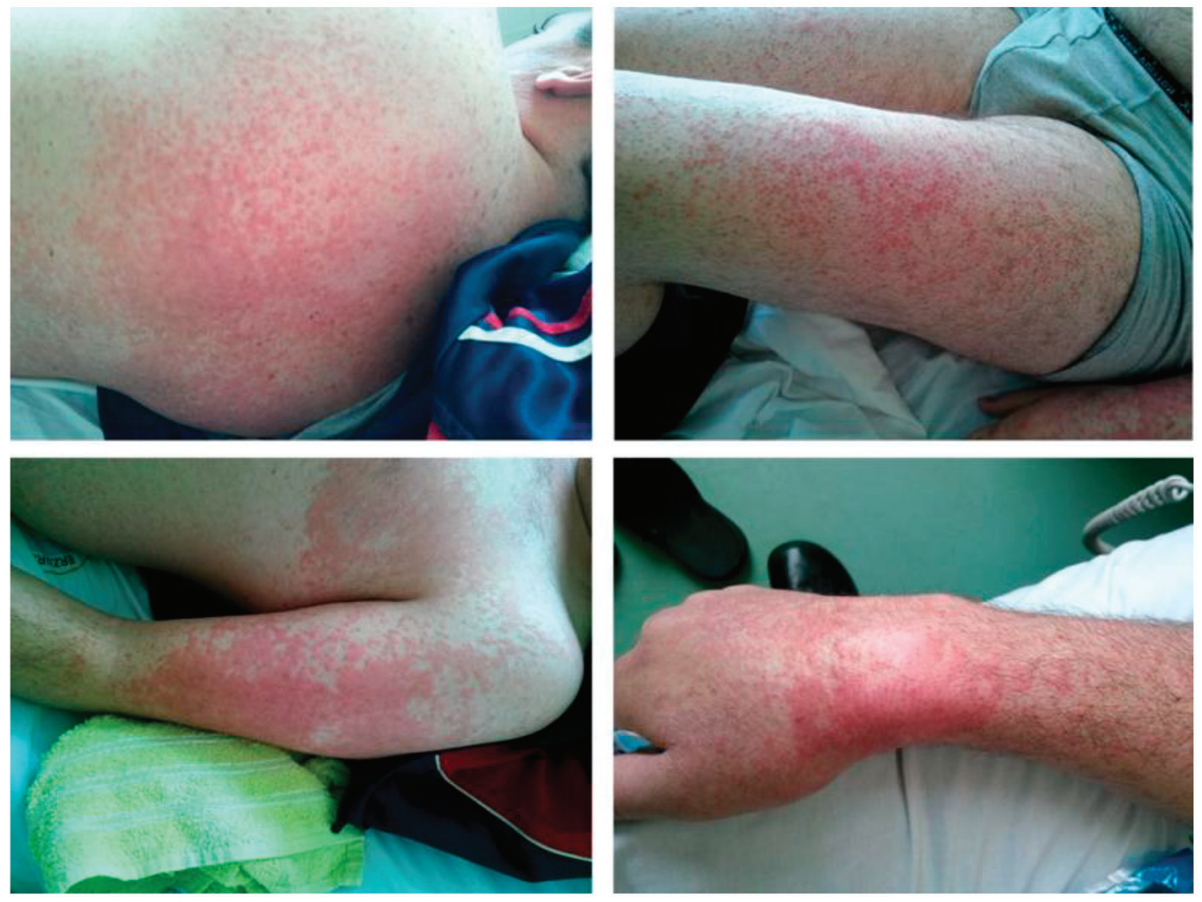

Figure 1. Maculo-papular rash on skin of leg, arm, wrist and torso.

biopsy was performed. Histopathologic examination results were consisted with drug related skin reaction (Fig. 2). According to these findings, LTG induced leukopenia, thrombocytopenia and rash were considered and LTG medication was ceased. After ceasing the LTG treatment skin lesions were gradually meliorated and blood cell counts were started to recover. Laboratory tests reached normal ranges at the end of the second week (Table 1).

\section{Discussion}

We present a case which had a combination of rare and clini-

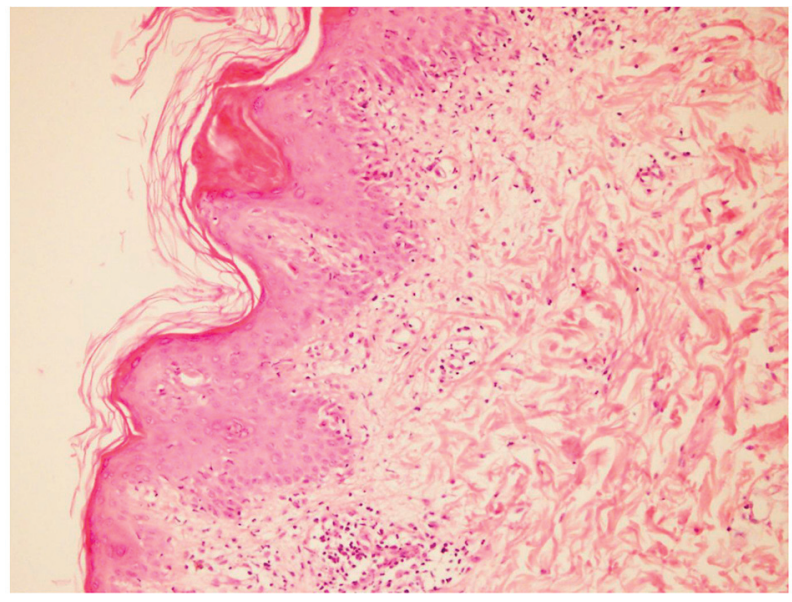

Figure 2. Microscopic view of skin biopsy specimen. Dermal inflammation with presence of hypereosinophilia. cally important adverse reactions associated with new started LTG treatment. Patient presented with febrile neutropenia and further progressed with rash. After investigation of other possible causes and establishing the correct diagnosis, medication of LTG ceased. Patient's hematologic abnormalities and skin lesions gradually recovered afterwards.

Existing antiepileptic drugs have considerable potential for concentration-dependent and idiosyncratic toxicity [3]. Most acute adverse reactions occur during the early stage of treatment, between 1 and 4 months [4]. Mild hematologic disorders can be encountered during treatment and they usually pose no threat to patient. In 11,316 patients who were included in a non-interventional observational cohort study, the hematological side effects among the other adverse effects were insignificant. According to previous reports, serious hematological side effects are rarely seen in patients treated with LTG [5]. There are approximately 40 cases of LTG induced leukopenia reported in the literature till now.

Table 1. Blood Cell Counts of the Patient Acquired at the Admission and Fourth, 10th and 18th Days After Ceasing LTG Medication

\begin{tabular}{llll}
\hline & $\begin{array}{l}\text { White blood } \\
\text { cell }\left(\times \mathbf{1 0}^{\mathbf{3}} / \boldsymbol{\mu L}\right)\end{array}$ & $\begin{array}{l}\text { Neutrophil } \\
\left(\times \mathbf{1 0}^{\mathbf{3}} / \boldsymbol{\mu L}\right)\end{array}$ & $\begin{array}{l}\text { Thrombocyte } \\
\left(\times \mathbf{1 0}^{\mathbf{3}} / \boldsymbol{\mu L}\right)\end{array}$ \\
\hline Admission & 2.32 & 1.2 & 116 \\
Fourth day & 2.39 & 1.45 & 183 \\
10th day & 4.6 & 1.64 & 163 \\
18th day & 4.9 & 1.84 & 197 \\
\hline
\end{tabular}

Normal ranges: WBC: $3.7-10.1 \times 10^{3} / \mu \mathrm{L}$; neutrophil: $1.4-6.5 \times 10^{3} / \mu \mathrm{L}$; thrombocyte: $150-450 \times 10^{3} / \mu \mathrm{L}$. 
Four reported cases were presented with leukopenia and thrombocytopenia only. Mechanisms responsible for LTG-related hematological complications are unknown. Risk factors thought to be associated with hematological effects include concomitant use of other antiepileptic drugs, and exceeding the recommended starting dose or the recommended rate of dose escalation [5].

Rash is seen more common than hematologic disorders as an adverse effect. Skin lesion characteristics are variable and lesions morphology also depends on the severity of the skin reaction. Based on severity of lesions, rash can be classified into four groups: 1) hypersensitivity reactions (e.g. fixed eruptions, pruritis, exanthematous, urticaria, and edema); 2) non-lifethreatening eruptions (e.g. photosensitivity, alopecia, drug-induced pigmentation); 3) severe and life-threatening conditions (e.g. erythema multiforme, Stevens-Johnson syndrome, toxic epidermal necrolysis, exfoliative dermatitis); and 4) exacerbation of other skin lesions (e.g. acne, psoriasis, seborrhea, and hyperhidrosis). There are approximately 200 of LTG related rash cases reported in literature [6]. Despite the necessity of drug withdrawal in case of leukopenia, average rate of withdrawal due to LTG rash is $2 \%$ [7]. Rash occurrence also linked with titration of LTG and concomitant use of valproic acid in previous studies [8].

\section{Conclusions}

Rash occurrence is often considered as hypersensitivity reaction. As in our case, it may follow hematologic complications. After stopping the medication, laboratory findings and skin lesions start to recover. It may take several days for blood counts to reach normal ranges and few weeks for skin lesions to diminish. We think that these side effects are associated with rapid increment of lamotrigine dose and concomitant use of valproic acid.

Clinician must be aware of serious side effects of LTG especially at the beginning of the treatment. Serious adverse effects such as leukopenia, thrombocytopenia and rash should be considered along with the other insignificant complaints such as nausea, dizziness and malaise.

\section{Conflict of Interest}

None.

\section{References}

1. Leach MJ, Marden CM, Miller AA. Pharmacological studies on lamotrigine, a novel potential antiepileptic drug: II. Neurochemical studies on the mechanism of action. Epilepsia. 1986;27(5):490-497.

2. French JA, Kanner AM, Bautista J, Abou-Khalil B, Browne T, Harden CL, Theodore WH, et al. Efficacy and tolerability of the new antiepileptic drugs II: treatment of refractory epilepsy: report of the Therapeutics and Technology Assessment Subcommittee and Quality Standards Subcommittee of the American Academy of Neurology and the American Epilepsy Society. Neurology. 2004;62(8):1261-1273.

3. Brodie MJ. Established anticonvulsants and treatment of refractory epilepsy. Lancet. 1990;336(8711):350-354.

4. Wong IC, Mawer GE, Sander JW. Adverse event monitoring in lamotrigine patients: a pharmacoepidemiologic study in the United Kingdom. Epilepsia. 2001;42(2):237244.

5. Mackay FJ, Wilton LV, Pearce GL, Freemantle SN, Mann RD. Safety of long-term lamotrigine in epilepsy. Epilepsia. 1997;38(8):881-886.

6. Lorberg B, Youssef NA, Bhagwagar Z. Lamotrigineassociated rash: to rechallenge or not to rechallenge? Int J Neuropsychopharmacol. 2009;12(2):257-265.

7. Messenheimer J, Mullens EL, Giorgi L, Young F. Safety review of adult clinical trial experience with lamotrigine. Drug Saf. 1998;18(4):281-296.

8. Choi H, Morrell MJ. Review of lamotrigine and its clinical applications in epilepsy. Expert Opin Pharmacother. 2003;4(2):243-251. 